\title{
Development of a Korean Culture-Friendly Olfactory Function Test and Optimization of a Diagnostic Cutoff Value
}

\author{
Jong-Gyun $\mathrm{Ha}^{1}$ (D) · Jinwon $\mathrm{Kim}^{1}$ (D) $\cdot$ Jae Sung Nam ${ }^{1}$ (D) $\cdot$ Jeong Jin Park ${ }^{1}$ (D) $\cdot$ Hyung-Ju Cho ${ }^{1,2,3}$ (iD \\ Joo-Heon Yoon ${ }^{1,2,3}$ (iD $\cdot$ Chang-Hoon $\mathrm{Kim}^{1,2,3,4}$ (D) \\ ${ }^{1}$ Department of Otorhinolaryngology, ${ }^{2}$ The Airway Mucus Institute, ${ }^{3}$ Korea Mouse Sensory Phenotyping Center, and ${ }^{4}$ Taste Research Center, \\ Yonsei University College of Medicine, Seoul, Korea
}

Objectives. Cultural familiarity and safety must be considered when assessing olfactory ability. The YSK olfactory function (YOF) test is a new olfactory function test using culturally familiar odorants to Koreans.

Methods. The YOF test comprises three subtests for threshold (T), discrimination (D), and identification (I). The identification test included eight universal and four Korean culture-friendly odorants, which were selected considering eight major functional groups. Data were obtained from 1,127 subjects over 19 years old. Subjects were classified as having normosmia $(n=542)$, hyposmia $(n=472)$, and anosmia $(n=113)$ by self-reported olfactory function. The YOF test and the Korean version of the Sniffin' stick test (KVSS-II) were performed on the same day in random order. Diagnostic cutoffs for anosmia and hyposmia were calculated using the Youden index (J).

Results. The mean values for each T/D/I subtest and the total TDI score were as follows: normosmia (T, 4.6 $\pm 2.3 ; \mathrm{D}, 8.6 \pm$ 2.1; I, 11.1 \pm 1.7 ; TDI score, 24.2 \pm 4.5 ); hyposmia (T, 3.3 \pm 2.2 ; D, $7.1 \pm 2.5$; I, 9.2 \pm 3.1 ; TDI score, $19.5 \pm 6.4$ ); and anosmia (T, $1.7 \pm 1.2$; D, $5.1 \pm 2.5$; I, 5.0 \pm 3.2 ; TDI score, $11.8 \pm 5.6$ ). The correlation coefficients between the YOF test and KVSS-II were $0.57,0.65,0.80$, and 0.86 for T, D, I, and the TDI score, respectively $(P<0.001)$. The diagnostic cutoffs were a TDI score $\leq 14.5(\mathrm{~J}=0.67)$ for anosmia and $14.5<$ TDI score $\leq 21.0(\mathrm{~J}=0.38)$ for hyposmia. The diagnostic efficacy of the YOF test (area under the curve [AUC], 0.88) was equivalent to that of the KVSS-II (AUC, 0.88; $P=0.843$; DeLong method).

Conclusion. The YOF test is a new olfactory test using safe and Korean culture-friendly odorants. It showed equivalent validity with the conventional olfactory function test. Furthermore, the YOF test provides information on the major functional groups of odorants, potentially enabling a more comprehensive interpretation for patients with olfactory disorders.

Keywords. Smell; Olfactory Perception; Culture; Odorants

\section{INTRODUCTION}

Olfaction determines the ability to detect various odors, including those associated with hazards such as spoiled food, gas

- Received May 13, 2020

Revised June 3, 2020

Accepted June 17, 2020

- Corresponding author: Chang-Hoon Kim

Department of Otorhinolaryngology, Yonsei University College of Medicine,

50-1 Yonsei-ro, Seodaemun-gu, Seoul 03722, Korea

Tel: +82-2-2228-3609, Fax: +82-2-393-0580

E-mail: entman@yuhs.ac leaks, and fires. Olfaction is related to quality of life [1] and survival [2]. It is also a prodromal marker for the early diagnosis of neurodegenerative and psychiatric disorders such as Parkinson disease, Alzheimer disease, and major depression [3-5]. Thus, there is an urgent need to accurately assess an individual's sense of smell appropriately. Many psychophysical olfactory tests have been developed and used in research and/or clinical settings; these evaluate one or more olfactory components of the detection threshold, discrimination, and identification. The detection threshold, discrimination, and odor identification are affected by low, middle, and high levels of olfactory processing, respectively,

Copyright $\odot 2020$ by Korean Society of Otorhinolaryngology-Head and Neck Surgery.

This is an open-access article distributed under the terms of the Creative Commons Attribution Non-Commercial License (https://creativecommons.org/licenses/by-nc/4.0)

which permits unrestricted non-commercial use, distribution, and reproduction in any medium, provided the original work is properly cited. 
and require different levels of cognition [6]. In particular, odor identification may require prior exposure to culturally-specific odor stimuli [7]. Therefore, many culture-friendly odor identification tests have been specifically developed or modified and validated [8].

The YSK olfactory function test (YOF test; Kimex Co., Suwon, Korea) is a new olfactory function test that uses culturally familiar odorants to Koreans. The 12 odorants of the identification test were selected to include all eight major chemical functional groups.

\section{MATERIALS AND METHODS}

\section{Study design and participants}

This prospective study was reviewed and approved by the Institutional Review Board of Severance Hospital, Seoul, Korea (IRB No. 4-2018-0861). Written consent was obtained from participants following oral and written explanations of the study objectives. All personally identifiable information was protected.

This study was conducted with adults (age $\geq 19$ years old) who underwent olfactory function tests including preoperative olfactory evaluation (i.e., septoplasty, turbinoplasty, etc.) in a tertiary medical institution in South Korea, from September 2018 to December 2019. Patients with cognitive dysfunction such as neurodegenerative disease and six patients with negative results for the ammonia test were excluded. Finally, total of 1,127 individuals (female, 493; male, 634) were enrolled. Participants were segregated into three groups by subjective self-assessment for olfactory function: normosmia group (542 participants), hyposmia group (472 participants), and anosmia group (113 participants). Each participant performed the YOF test and the Korean version of the Sniffin' stick test (KVSS-II) on the same day in random order with 3 minutes' interval between the two tests to prevent olfactory adaptation. In order to confirm whether the odorants were suitable for the evaluation of the sense of smell in the normal people, the rate of correct answer for each step of the discrimination and identification test was checked for the normosmia

\section{H I I G}

- The YSK olfactory function (YOF) test consists of threshold, discrimination, and odor identification tests.

- The YOF test adopted phenylethyl alcohol, a safe chemical, as an odorant for the threshold test.

- The YOF test used culturally familiar odors to Koreans and was designed to include eight major functional groups.

- The diagnostic cutoff of the YOF test was $\leq 14.5$ for anosmia and $\leq 21.0$ for hyposmia.

- The YOF test correlated well with the Korean version of the Sniffin' stick test and showed equivalent diagnostic efficacy.

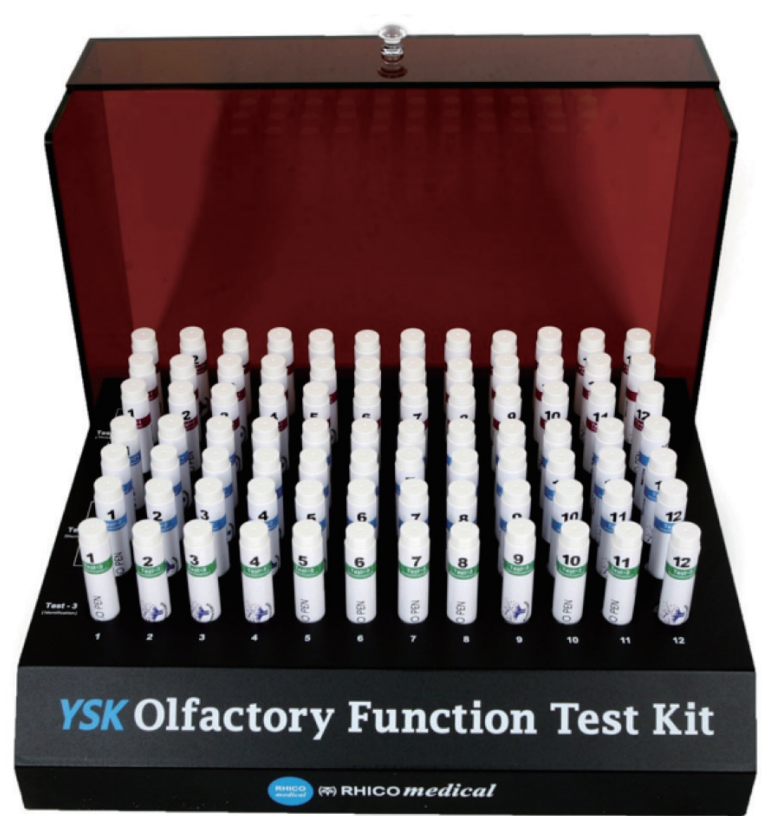

Fig. 1. The YSK olfactory function test is composed of three subtests of threshold, discrimination, and identification, which each have 12-point scales.

group. Optimized cutoff values for the YOF test for anosmia vs. hyposmia vs. normosmia were calculated.

\section{YOF test protocol}

The YOF test was designed considering the chemical and structural characteristics of the odorants and cultural experience and safety of participants. The YOF test included three subtests, namely, threshold, discrimination, and identification tests. Score range of the YOF subtests were 1-12 for threshold test, 0-12 for discrimination test and identification test, and 1-36 for total TDI score. The commercially available YOF test provided all odorants as felt-tip pens (Fig. 1). The test procedure required covering the subject's eyes, placing the pens $2 \mathrm{~cm}$ away from the nostrils, and subsequently smelling each pen for 3 seconds. All the YOF test procedures were performed in the olfactory function test room equipped with an air ventilation hood.

\section{Odor thresholds test ( $T$ )}

The odorant for the threshold test was 2-phenylethyl alcohol (PEA), a pleasant, rose-like odorant having minimal trigeminal nerve stimulation [9]. PEA is one of the most frequently selected test odorants for odor threshold tests. Our protocol required dilution of PEA in a geometric series consisting of 12 steps, with a dilution ratio of $1: 2$ beginning with $10 \%(\mathrm{v} / \mathrm{v})$ and using dipropylene glycol (DPG) as a solvent. The test method followed a three alternative forced-choice and seven-reversal initially ascending single-staircase procedure, as described by Doty et al. [10]. When starting the threshold test, the subject first smells the strongest concentration of PEA pen to recognize the rose-like 
odor of PEA. And then, a triplet of odorant pens was presented to each subject in a randomized order, with only one pen containing PEA at a particular dilution and the others containing only solvent (DPG). Subjects were directed to choose a rosescented pen from the triplet. If an incorrect response was given in any trial beginning with the lowest concentration, the staircase was moved upward until the correct pen was detected at a given concentration in two consecutive trial sets. If subjects gave the correct answer twice in a row, the process went down onestep, and they were directed to choose the correct smell again. The test was conducted until 7 turning points occurred. The geometric mean of the last 4 staircase reversal points in a total of 7 was used as the estimated detection threshold value.

\section{Odor discrimination test (D)}

The odor discrimination test incorporated the triplet forced test to assess capability for distinguishing different odorants. The 12 pen triplets for each step included one pen containing one target odorant and two others containing a non-target odorant (Table 1). The target and non-target odorants were set to a similar intensity for the test. Since the chemical complexity of the odorants can affect the sense of smell, the odorants of the discrimination test used a single chemical odorant rather than a mixture [11]. Subjects were directed to select one from each pen triplet. Each step was designed to be easily distinguishable by at least two of three average individuals.

\section{Odor identification test (I)}

In the preliminary study to select odorants for identification test, 113 (male, 71; female, 42; mean age, 38.5 years) subjects with normal olfactory function underwent a test with 32 candidate odorants. These odorants were presented as liquid in brown bottles $3 \mathrm{~cm}$ away from subjects. Subjects smelled the odorants by

Table 1. Odorants used in the YOF discrimination test and the correct answer rates for the normosmia group

\begin{tabular}{llclc}
\hline $\begin{array}{c}\text { Material } \\
\text { number }\end{array}$ & \multicolumn{1}{c}{$\begin{array}{c}\text { Target } \\
\text { odorant }\end{array}$} & $\begin{array}{c}\text { Material } \\
\text { number }\end{array}$ & $\begin{array}{c}\text { Non-target } \\
\text { odorant }\end{array}$ & $\begin{array}{c}\% \\
\text { Correct }\end{array}$ \\
\hline 1 & Aldehyde C-14 & $1-1$ & Ethyl caproate & 66.1 \\
2 & Aldehyde C-16 & $2-2$ & Eucalyptol & 69.3 \\
3 & Anisaldehyde & $3-3$ & Floralozone & 72.9 \\
4 & Anethol & $4-4$ & Hexyl acetate & 67.4 \\
5 & Allyl cyclohexyl & $5-5$ & Hexyl salicylate & 82.1 \\
& propionate & & & \\
6 & Benzyl acetate & $6-6$ & Isobornyl acetate & 68.3 \\
7 & Styrallyl acetate & $7-7$ & Para-cresol methyl & 76.6 \\
& & & ether & \\
8 & Citronellyl acetate & $8-8$ & Methyl anthranilate & 66.1 \\
\hline 9 & Cyclaprop & $9-9$ & Methyl benzoate & 78.0 \\
10 & Damascone delta & $10-10$ & Methyl cedryl ketone & 65.6 \\
11 & Decalactone gamma & $11-11$ & DMBC butyrate & 86.7 \\
12 & Manzanate & $12-12$ & Ligustral & 88.1 \\
\hline
\end{tabular}

YOF, YSK olfactory function. cupping their hands above the bottles and wafting the air toward their faces. Fourteen odorants showed results with $>90 \%$ correct answers in the preliminary test. Finally, considering cultural familiarity and including eight major chemical functional groups, 12 odorants were selected. The YOF identification test comprised eight universal odorants and four Korean culturefriendly odorants. In addition, it covered eight major chemical functional groups for odorants, namely, ketone, terpene, aldehyde, aromatic, alcohol, ester, acid, and amine (Table 2).

The odor identification test was designed as a multiple forced choice from four choices (Table 3). All odorants of identification test were presented as a felt tip pen. Subjects had to make one choice from four descriptors after smelling the pen for $3 \mathrm{sec}-$ onds. From step to step, there was 30 seconds of interval to prevent olfactory fatigue.

\section{Test protocol for KVSS-II}

KVSS-II is a Korean version of the Sniffin' stick test-an olfactory function test frequently administered worldwide [12]. The test method used here was identical to the original Sniffin' stick test [13]. The KVSS-II includes 16 stages of an n-butanol threshold test, 16 of a discrimination test, and 16 of an identification test. The threshold test incorporates 1:2 dilutions of n-butanol, beginning with $4 \%$ (the highest concentration), identical to the Sniffin' stick test.The KVSS-II identification test includes 16 unique odorants specifically modified to be familiar to Koreans. Validity of the KVSS-II test has been accepted in comparisons with other olfactory tests such as cross-cultural smell identification test and T\&T olfactometer $[14,15]$. The KVSS-II TDI score ranged from 1 to 20 for anosmia, 20.25-27 for hyposmia, and $\geq 27.25$ for normosmia [15].

\section{Optimization of cutoff criteria for the YOF test Cutoff for anosmia}

All participants were divided into binary groups of anosmic (anosmia group, $\mathrm{n}=113$ ) and non-anosmic (hyposmia+normosmia group, $n=1,014)$ subjects. The receiver operating characteristic (ROC) curve of TDI scores of the YOF test and KVSS-II for an-

\section{Table 2. Composition of odorants for the YOF identification test}

\begin{tabular}{lll}
$\begin{array}{c}\text { Major functional } \\
\text { group }\end{array}$ & \multicolumn{1}{c}{$\begin{array}{c}\text { Universal } \\
\text { odorant }\end{array}$} & $\begin{array}{c}\text { Korean culture-friendly } \\
\text { odorant }\end{array}$ \\
\hline $\begin{array}{l}\text { Ketone } \\
\text { Terpene }\end{array}$ & $\begin{array}{l}\text { Spearmint } \\
\text { Baby powder }\end{array}$ & Oriental medicine \\
$\begin{array}{l}\text { Aldehyde } \\
\text { Aromatic }\end{array}$ & $\begin{array}{l}\text { Cinnamon } \\
\text { Chocolate }\end{array}$ & \\
Alcohol & Medicated patch & Marinated grilled beef \\
Ester & Ashes & Korean red ginseng \\
Acid & Peach & \\
Amine & Naphthalene & Scorched rice \\
\hline
\end{tabular}

YOF, YSK olfactory function. 
Table 3. Odorants used in the YOF identification test, their distractors, and the correct answer rates for the normosmia group

\begin{tabular}{|c|c|c|c|c|c|}
\hline Number & & Odor & distractor & & $\%$ Correct \\
\hline 1 & Baby powder ${ }^{a)}$ & Apple & Curry & Chocolate & 96.3 \\
\hline 2 & Strawberry & Rose & Cinnamon ${ }^{a)}$ & Lemon & 80.7 \\
\hline 3 & Ginseng & Watermelon & Peacha) & Peanut & 90.8 \\
\hline 4 & Prune & Melon & Scorched rice ${ }^{a)}$ & Acacia flowers & 97.2 \\
\hline 5 & Spearminta) & Apple & Orange & Tree & 93.6 \\
\hline 6 & Chocolate $^{\text {a) }}$ & Mugwort & Garlic & Grape & 96.3 \\
\hline 7 & Strawberry & Grape & Oriental medicine $e^{a)}$ & Kimchi & 98.6 \\
\hline 8 & Medicated patcha) & Cherry & Chocolate & Rose & 96.3 \\
\hline 9 & Cotton candy & Honey & Korean red ginseng ${ }^{a)}$ & Kimchi & 94.0 \\
\hline 10 & Grapefruit & Naphthalene ${ }^{\text {a) }}$ & Coffee & Rose & 87.2 \\
\hline 11 & Corn & Lemon & Marinated grilled beefa) & Soap & 95.0 \\
\hline 12 & Melon & Ginger & Banana & Ashes $^{\text {a) }}$ & 97.2 \\
\hline
\end{tabular}

YOF, YSK olfactory function.

a)Test odorants.

osmia were plotted. The optimized cutoff for anosmia was the point with the maximum Youden index [16].

\section{Cutoff for hyposmia}

After calculating the cutoff value of YOF test for anosmia, ROC curve TDI scores of the YOF test and KVSS-II for hyposmia were plotted for non-anosmic subjects (hyposmia group, $n=472$; normosmia group, $n=542$ ). The optimized cutoff value for hyposmia was determined using the Youden index.

\section{Statistical analyses}

All continuous variables are reported as mean \pm standard deviation (SD) and categorical variables as $n(\%)$. Comparisons of normal distribution data among the groups were performed using chi-square test for categorical data and one-way analysis of variance (ANOVA) for continuous variables Possible differences in the results of the YOF test and KVSS-II were determined via a post hoc Tukey test.

To determine the cutoff values for anosmia and hyposmia in the YOF test, the ROC curves were plotted. Optimal cutoff values for the anosmia and hyposmia were determined using the Youden index ( $\mathrm{J}$; sensitivity+specificity-1), which has a value between 0 and 1 . The highest $\mathrm{J}$ value is considered the optimal cutoff. The area under the curve (AUC) was also calculated to evaluate the diagnostic efficacy of the YOF test. Statistical comparison of AUC of ROC curve between the YOF test and KVSSII were performed according to the DeLong method [17].

The Pearson coefficient was calculated to determine the correlation of the YOF test and KVSS-II results. In addition, because the scales of the YOF test and KVSS-II are different, the score ratios (0 to 1$)$ for each score scale was compared using an independent samples $t$-test. IBM SPSS ver. 25.0 (IBM Corp., Armonk, NY, USA) was for statistical analysis of independent samples $t$-test, one-way ANOVA, Mann-Whitney $U$-test, logistic regression test, and Pearson coefficient correlation. MedCalc software ver. 14.8.1 (MedCalc Software, Mariakerke, Belgium) was used to assess the cutoff points of TDI score of the YOF test and KVSS-II for anosmia and hyposmia and compare their AUC. $P<0.05$ indicated statistical significance.

\section{RESULTS}

\section{Demographic data and mean olfactory function test scores}

The demographic data and mean values for each T/D/I subtest and the TDI score from each group are presented in Table 4. The sex ratios (male-to-female) within each group were significantly different (normosmia group, 335:207; hyposmia group, 245:227; anosmia group, 54:59; $P<0.001$ with the Pearson chi-square test). The rate of anosmia was higher in females, but this difference was not significant $(P=0.069)$. Mean age was significantly higher in the anosmia group than in the other groups (normosmia group, $47.0 \pm 16.9$ years; hyposmia group, $49.6 \pm 16.3$ years; anosmia group, $52.9 \pm 14.1$ years; $P<0.001$, with one-way ANOVA). According to the post hoc Tukey analysis, significant differences were found between the mean age of the normosmia group and that of the hyposmia $(P=0.031)$ and anosmia groups $(P<0.001)$, but not between the mean age of the hyposmia group and the anosmia group $(P=0.131)$. The mean values of each T/D/I subtest and the total TDI scores among the normosmia, hyposmia, and anosmia groups were significantly different in both the YOF test and KVSS-II $(P<0.001)$.

\section{Comparison between the YOF test and KVSS-II in the normosmia group}

The score distributions (Fig. 2A-D) and olfactory function by age (Fig. 2E-H) of each T/D/I subtest for threshold, discrimination, and identification, as well as the total TDI score, are shown in Fig. 2. The results for threshold $\left(\beta=-0.025, \mathrm{R}^{2}=0.031, P<0.001\right)$, discrimination $\left(\beta=-0.046, \mathrm{R}^{2}=0.088, P<0.001\right)$, identification 

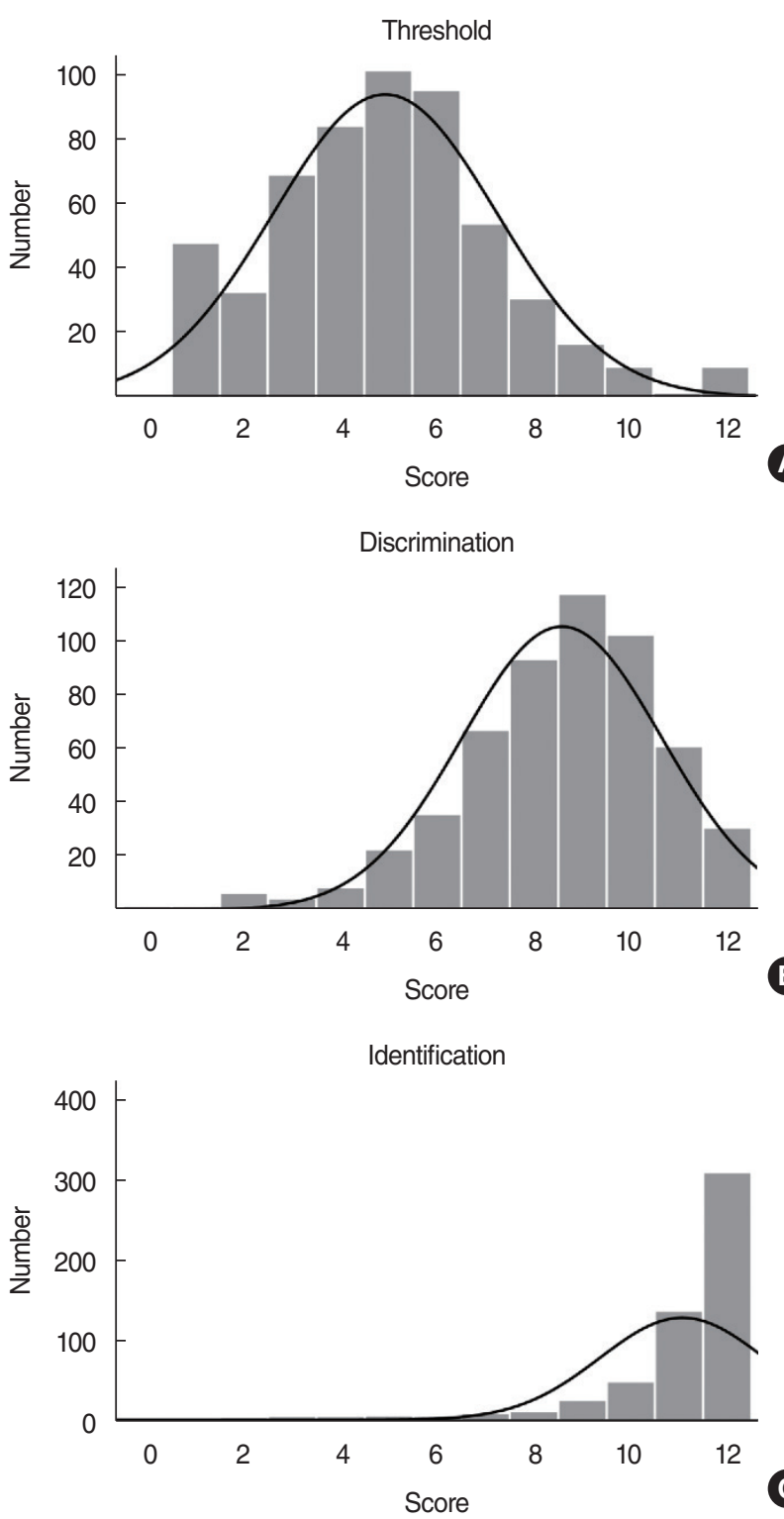

Total TDI score

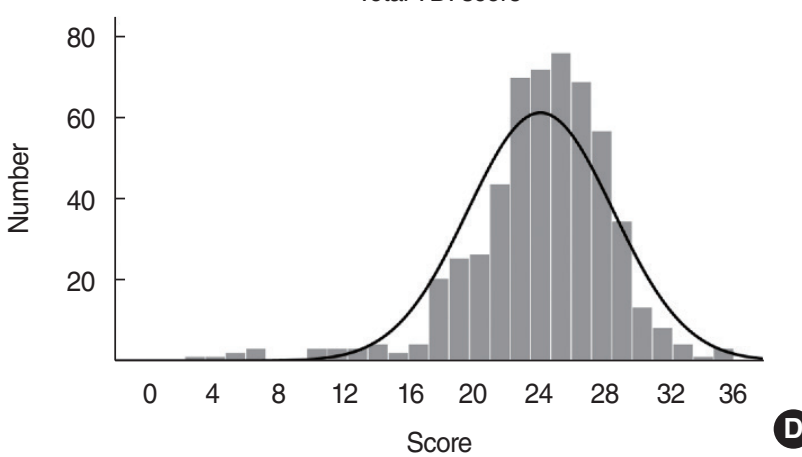

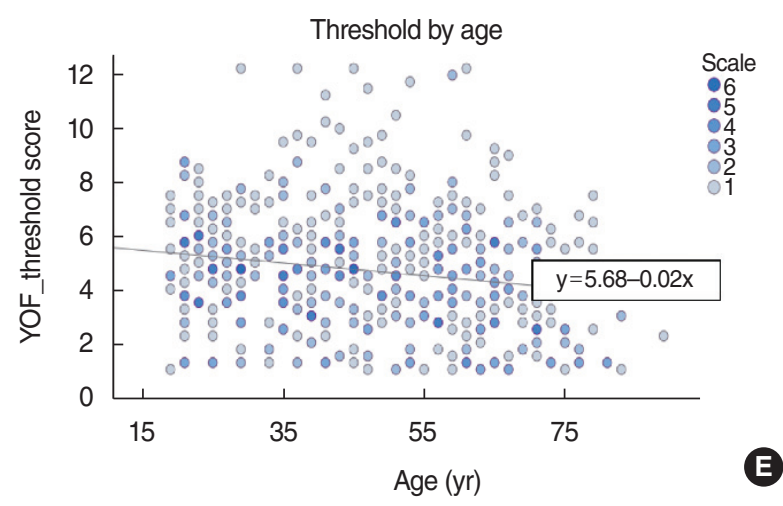
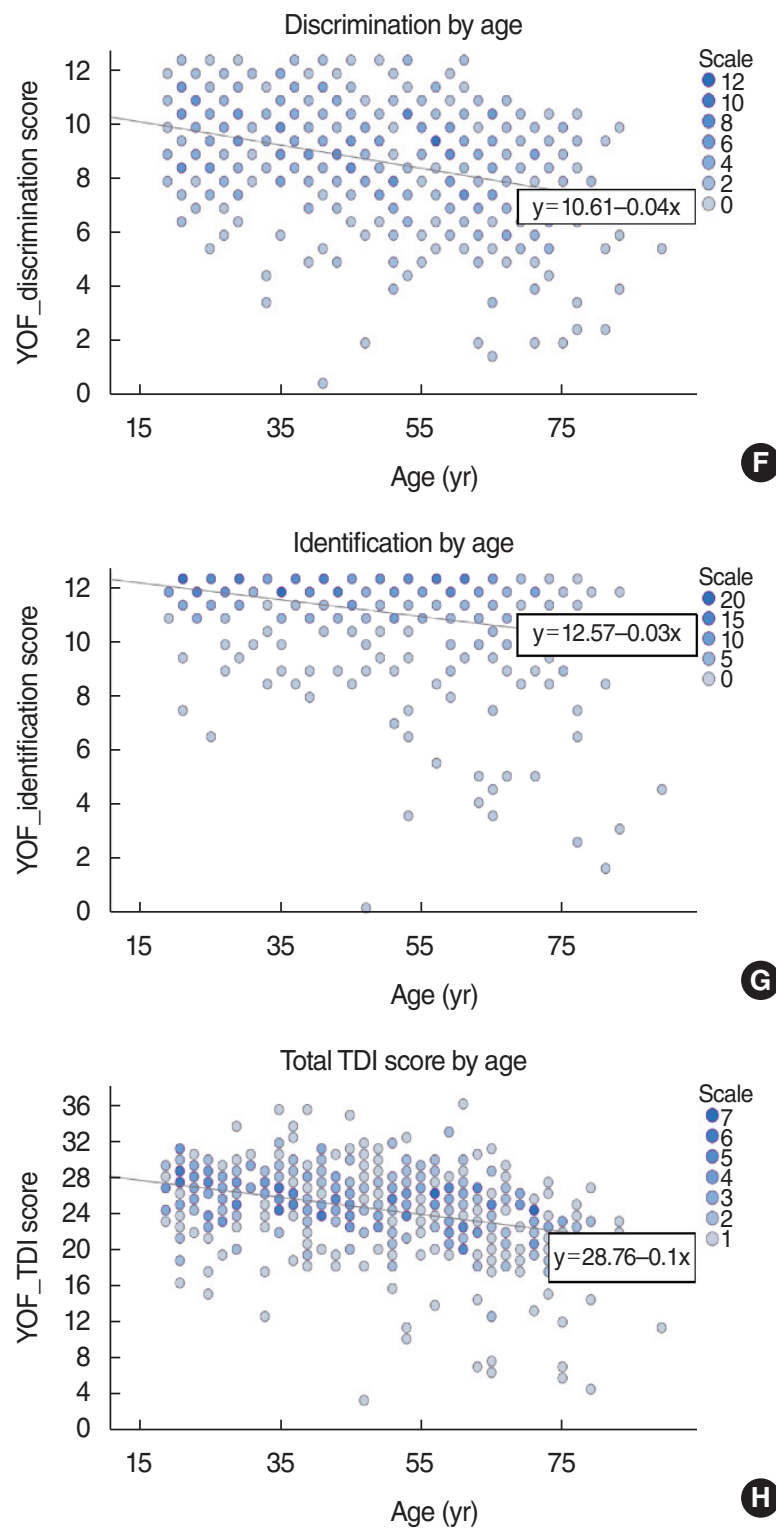

Fig. 2. Score distributions and scatter plot by age of the total TDI score and each T/D/l subtest. The results of the YSK olfactory function (YOF) test of the normosmia group showed grossly normal distributions ( $A, B, D)$ except for the identification test (C, intentionally skewed to the right). The horizontal scale of the threshold graph $(A)$ is expressed in units of 1 point for simple visualization. For total TDI score and all results of each $\mathrm{T} / \mathrm{D} / \mathrm{I}$ subtest, olfactory function significantly decreased with increasing age of the subjects $(\mathrm{E}-\mathrm{H} ; \mathrm{P}<0.001$, respectively). T, threshold; $\mathrm{D}$, discrimination; I, identification. 
$\left(\beta=-0.046, R^{2}=0.060, P<0.001\right)$, and totalTDI score $(\beta=-0.115$, $\left.\mathrm{R}^{2}=0.081, P<0.001\right)$ consistently showed significant negative correlations with age in the logistic regression analysis. For greater clarity, the distribution of the YOF test and KVSS-II scores (totalTDI score and T/D/I subtest scores) in the normosmia group were graphically expressed through the score ratio (0 to 1 , test score/score scale) (Supplementary Fig. 1).

\section{Threshold}

The threshold score of the YOF test followed a normal distribution (Fig. 2A). The mean T value of the YOF test was $4.6 \pm 2.3$ on a 12-point scale, whereas that for the KVSS-II was $6.8 \pm 2.6$ on a 16-point scale in the normosmia group (Table 4). There was a significant difference in the mean score ratio of the threshold test between the YOF test (mean, 0.38; standard error of the mean [SEM], 0.01) and the KVSS-II (mean, 0.42; SEM, 0.01) $(P<0.001)$ (Supplementary Fig. 1). This statistical difference was caused by differences in the content of each olfactory function test (e.g., test odorant, concentration, score scales, etc.).

\section{Discrimination}

The percentage of correct answers for each triplet ranged from $65.6 \%$ to $88.1 \%$ (Table 1 ). The mean D score of the YOF test for the normosmia group was $8.6 \pm 2.1$ on a 12-point scale, whereas that for the KVSS-II was $11.5 \pm 2.7$ on a 16 -point scale (Table 4). There was no significant difference in the mean score ratio of the discrimination test between the YOF test (mean, 0.71; SEM, $0.01)$ and the KVSS-II (mean, 0.72; SEM, 0.01) $(t=0.23$, $P=0.820$ ) (Supplementary Fig. 1).

\section{Identification}

The percentage of correct identifications for the universal odorants ranged from $80.7 \%$ to $97.2 \%$ (mean, $92.3 \%$; SD, $5.4 \%$ ) and that for the Korean culture-friendly odorants ranged from $94.0 \%$ to $98.6 \%$ (mean, $96.2 \%$; SD, $2.1 \%$ ) (Table 3). However, there was no significant difference in the mean percentage of correct identifications between the universal odorants and Korean culturefriendly odorants $(P=0.214$, Mann-Whitney $U$-test). The mean I score on the YOF test for the normosmia group was $11.1 \pm 1.7$ on a 12-point scale, whereas that for the KVSS-II was $12.1 \pm 2.3$ on a 16-point scale (Table 4). For the identification test, the YOF test (mean, 0.92; SEM, 0.01) showed a significantly higher mean score ratio than the KVSS-II (mean, 0.76; SEM, 0.01; $P<0.001$ ) (Supplementary Fig. 1).

\section{Total TDI score}

The totalTDI score is the sum of the scores of threshold, discrimination, and identification subtests. The mean total TDI score of the YOF test was $24.2 \pm 4.5$ on a 36 -point scale, whereas the mean totalTDI score of the KVSS-II was $30.4 \pm 5.9$ on a 48 -point scale (Table 4). There was a significant difference in the mean score ratio of the total TDI score between the YOF test (mean, 0.67;
Table 4. Demographic data and descriptive statics for the YOF test and the KVSS-II by self-assessed olfactory function

\begin{tabular}{|c|c|c|c|c|c|}
\hline Variable & $\begin{array}{c}\text { Normosmia } \\
(n=542)\end{array}$ & $\begin{array}{c}\text { Hyposmia } \\
(n=472)\end{array}$ & $\begin{array}{l}\text { Anosmia } \\
(n=113)\end{array}$ & $P$-value & $\begin{array}{c}\text { Post hoc } \\
\text { test }\end{array}$ \\
\hline \multicolumn{6}{|l|}{ Sex } \\
\hline Male:female & $335: 207$ & 245:227 & $54: 59$ & $<0.001^{\text {a) }}$ & \\
\hline Age (yr) & $47.0 \pm 16.9$ & $49.6 \pm 16.3$ & $52.9 \pm 14.1$ & $<0.001^{\text {b) }}$ & $\begin{array}{l}P 1=0.031 \\
P 2<0.001 \\
P 3=0.131\end{array}$ \\
\hline \multicolumn{6}{|l|}{ YOF test } \\
\hline TDI score & $24.2 \pm 4.5$ & $19.5 \pm 6.4$ & $11.8 \pm 5.6$ & $<0.001^{\text {b) }}$ & $\begin{array}{l}P 1<0.001 \\
P 2<0.001 \\
P 3<0.001\end{array}$ \\
\hline T & $4.6 \pm 2.3$ & $3.3 \pm 2.2$ & $1.7 \pm 1.2$ & $<0.001^{\text {b) }}$ & $\begin{array}{l}P 1<0.001 \\
P 2<0.001 \\
P 3<0.001\end{array}$ \\
\hline $\mathrm{D}$ & $8.6 \pm 2.1$ & $7.1 \pm 2.5$ & $5.1 \pm 2.5$ & $<0.001^{\text {b) }}$ & $\begin{array}{l}\mathrm{P} 1<0.001 \\
\mathrm{P} 2<0.001 \\
\mathrm{P} 3<0.001\end{array}$ \\
\hline I & $11.1 \pm 1.7$ & $9.2 \pm 3.1$ & $5.0 \pm 3.2$ & $<0.001^{\mathrm{b})}$ & $\begin{array}{l}P 1<0.001 \\
P 2<0.001 \\
P 3<0.001\end{array}$ \\
\hline \multicolumn{6}{|l|}{ KVSS-II } \\
\hline TDI score & $30.4 \pm 5.9$ & $24.3 \pm 8.2$ & $14.6 \pm 6.7$ & $<0.001^{\text {b) }}$ & $\begin{array}{l}P 1<0.001 \\
P 2<0.001 \\
P 3<0.001\end{array}$ \\
\hline $\mathrm{T}$ & $6.8 \pm 2.6$ & $5.0 \pm 2.8$ & $2.5 \pm 1.9$ & $<0.001^{\text {b) }}$ & $\begin{array}{l}P 1<0.001 \\
P 2<0.001 \\
P 3<0.001\end{array}$ \\
\hline $\mathrm{D}$ & $11.5 \pm 2.7$ & $9.4 \pm 3.1$ & $6.2 \pm 2.9$ & $<0.001^{\text {b) }}$ & $\begin{array}{l}P 1<0.001 \\
P 2<0.001 \\
P 3<0.001\end{array}$ \\
\hline I & $12.1 \pm 2.3$ & $10.0 \pm 3.7$ & $5.7 \pm 3.1$ & $<0.001^{\text {b) }}$ & $\begin{array}{l}P 1<0.001 \\
P 2<0.001 \\
P 3<0.001\end{array}$ \\
\hline
\end{tabular}

Values are presented as mean \pm standard deviation. $\mathrm{P} 1$, the difference between the normosmia group and the hyposmia group; P2, the difference between the normosmia group and the anosmia group; P3, the difference between the hyposmia group and the anosmia group. The KVSS-II score for each TDI subtest is shown.

YOF, YSK olfactory function; KVSS-II, Korean version of the Sniffin' stick test; T, threshold; D, discrimination; I, identification.

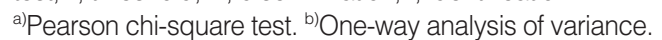

SEM, 0.01) and the KVSS-II (mean, 0.63; SEM, 0.01; $P<0.001$ ) (Supplementary Fig. 1).

\section{Optimized diagnostic cutoff of the YOF test and comparison with the KVSS-II}

Fig. 3 shows the ROC curve of the YOF test and the KVSS-II for anosmia and hyposmia.

\section{Cutoff of total TDI score for anosmia}

The AUC of the ROC curve of the total TDI score of the YOF test for anosmia was 0.88 (95\% confidence interval [CI], 0.85$0.89)$ (Fig. 3A). The AUC and the Youden J score (0.67) were maximized when the cutoff point was set to a TDI score of 14.5. 

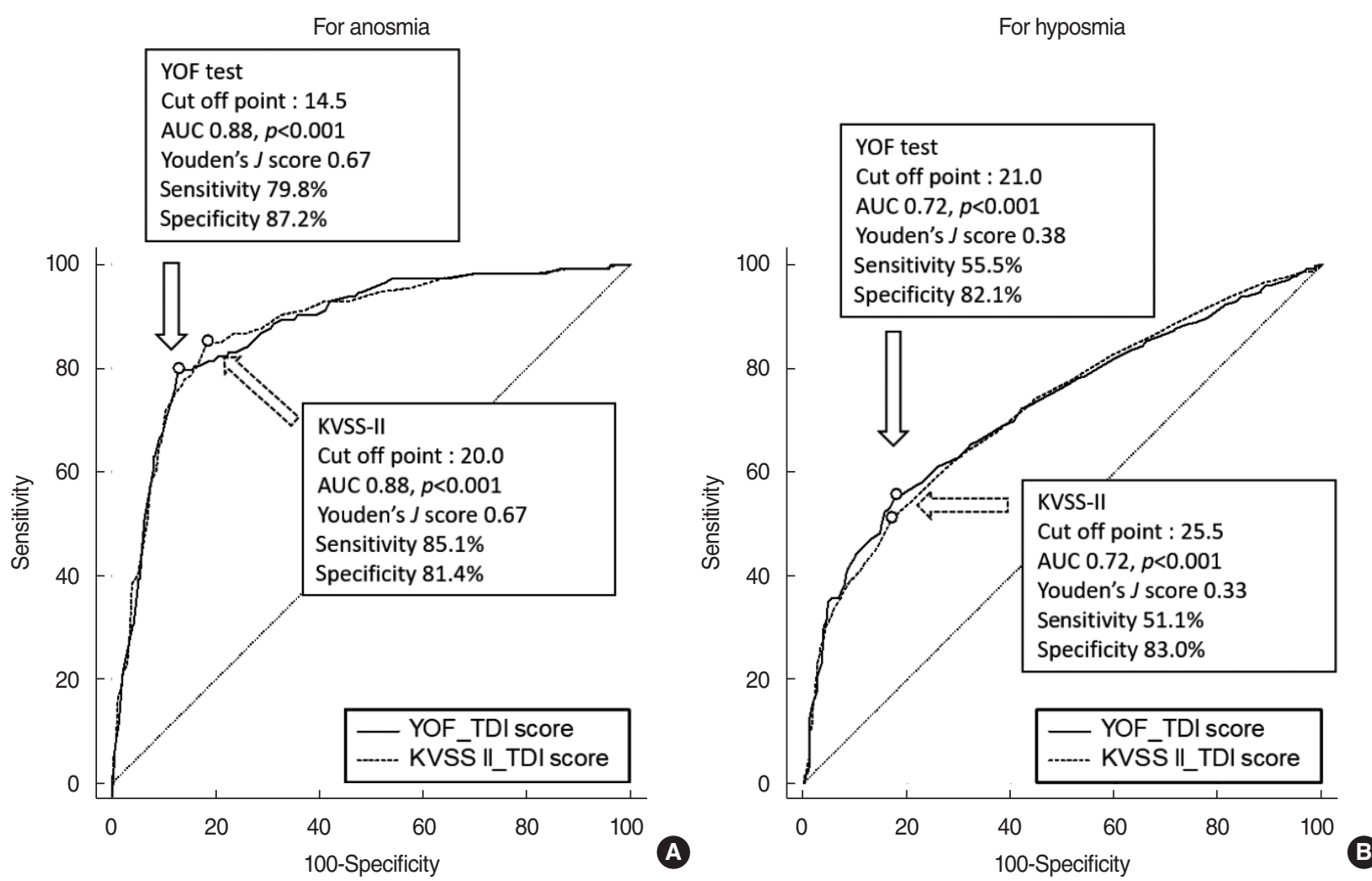

Fig. 3. The receiver operating characteristic (ROC) curve for the total TDI scores of the YSK olfactory function (YOF) test and Korean version of the Sniffin' stick test (KVSS-II) to predict anosmia (A) and hyposmia (B). The diagnostic cutoffs of the YOF test calculated through the Youden index were as follows: anosmia (TDI score $\leq 14.5$ : AUC, 0.88; $P<0.001$; Youden J score: 0.67; sensitivity, 79.8\%; specificity, 87.2\%) and hyposmia (14.5<TDI score $\leq 21.0$; AUC, 0.72; P<0.001; Youden J score, 0.38; sensitivity, 55.5\%; specificity, 82.1\%). The diagnostic cutoffs of the KVSS-II calculated through the Youden index were as follows: anosmia (TDI score $\leq 20.0 ;$ AUC, 0.88; $P<0.001$; Youden J score, 0.67; sensitivity, 85.1\%; specificity, 81.4\%) and hyposmia (20.0<TDI score $\leq 25.5$; AUC, 0.72; $P<0.001$, Youden J score, 0.33; sensitivity, 51.1\%; specificity, $83.0 \%)$. There was no significant difference in the AUC for anosmia $(P=0.843)$ and hyposmia $(P=0.902)$ between the YOF test and the KVSS-II. Points corresponding to the Youden $\mathrm{J}$ score were marked as circles on the ROC curve. T, threshold; D, discrimination; I, identification; AUC, area under the curve.

Sensitivity and specificity were $79.8 \%$ and $87.2 \%$, respectively. There was no significant difference in the AUC of the ROC curve for anosmia between the YOF test and the KVSS-II (AUC, 0.88 ; 95\% CI, 0.86-0.90; $P=0.843$ ).

\section{Cutoff of total TDI score for hyposmia}

The AUC of the ROC curve of the total TDI score of the YOF test for hyposmia was 0.72 (95\% CI, 0.69-0.75) (Fig. 3B). The optimized cutoff of the TDI score of YOF test for hyposmia was 21.0, and the Youden J score was 0.38. Sensitivity and specificity were $55.5 \%$ and $82.1 \%$, respectively. There was also no significant difference in the AUC of the ROC curve for anosmia between the YOF test and the KVSS-II (AUC, 0.72; 95\% CI, $0.69-0.75 ; P=0.902)$.

\section{Correlations between the YOF test and the KVSS-II}

We found positive correlations for each T/D/I subtest and the total TDI scores between the YOF test and the KVSS-II (Fig. 4). The Pearson correlation coefficient $(r)$ between the YOF test and the KVSS-II was $r=0.57$ for the T test, $r=0.65$ for the D test, $r=0.80$ for the I test, and $r=0.86$ for the totalTDI score $(P<0.001$, respectively). The total TDI scores showed a higher correlation between the YOF test and the KVSS-II than any of the T/D/I subtest scores.

\section{DISCUSSION}

We developed a new olfactory test suitable for evaluating olfactory function in Koreans. Furthermore, the odorants of the identification test of the YOF test were constructed considering their major chemical functional groups. The test adopted a 12-point scale for threshold detection, discrimination, and odor identification, similar to other modern psychophysical olfactory function test batteries [8]. According to our results, the YOF test was closely correlated with the KVSS-II and also showed good performance in distinguishing Koreans' olfactory function into normosmia, hyposmia, and anosmia. 

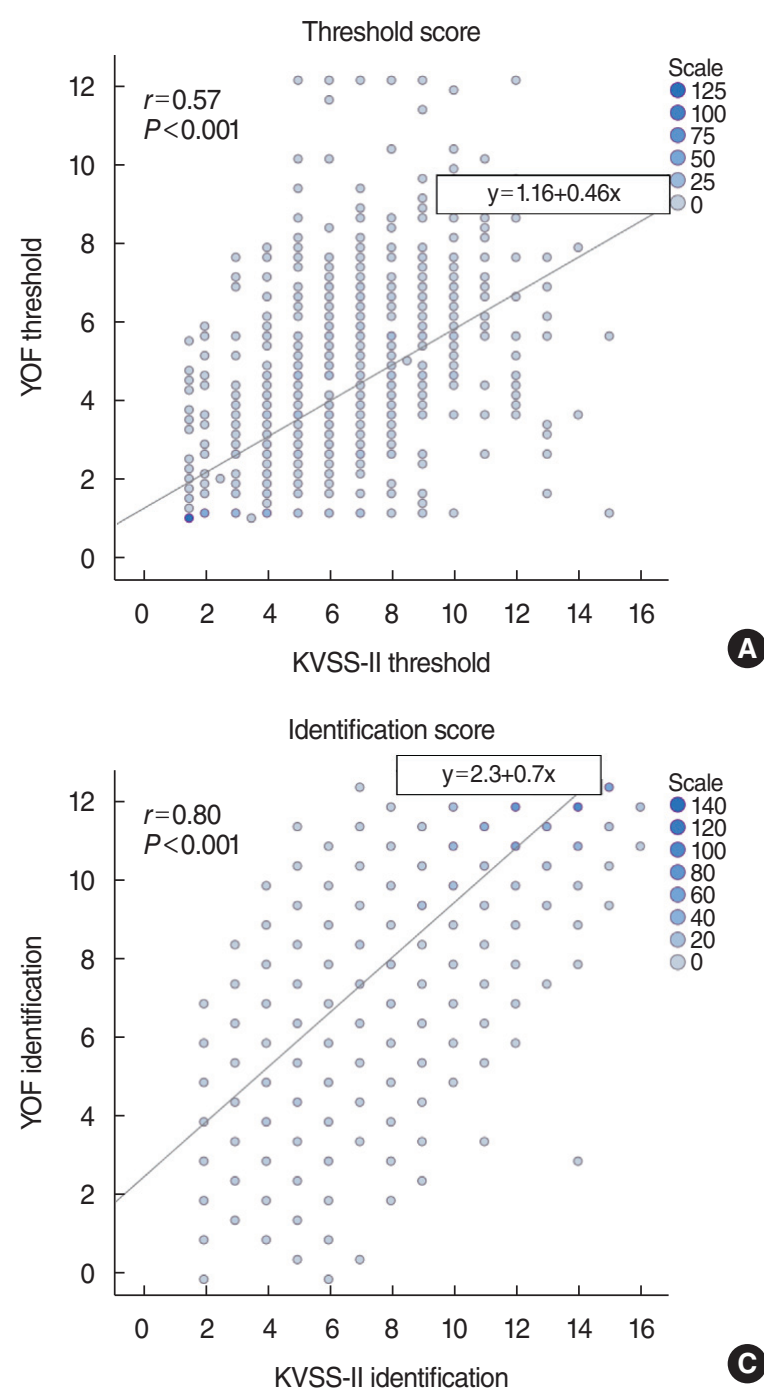
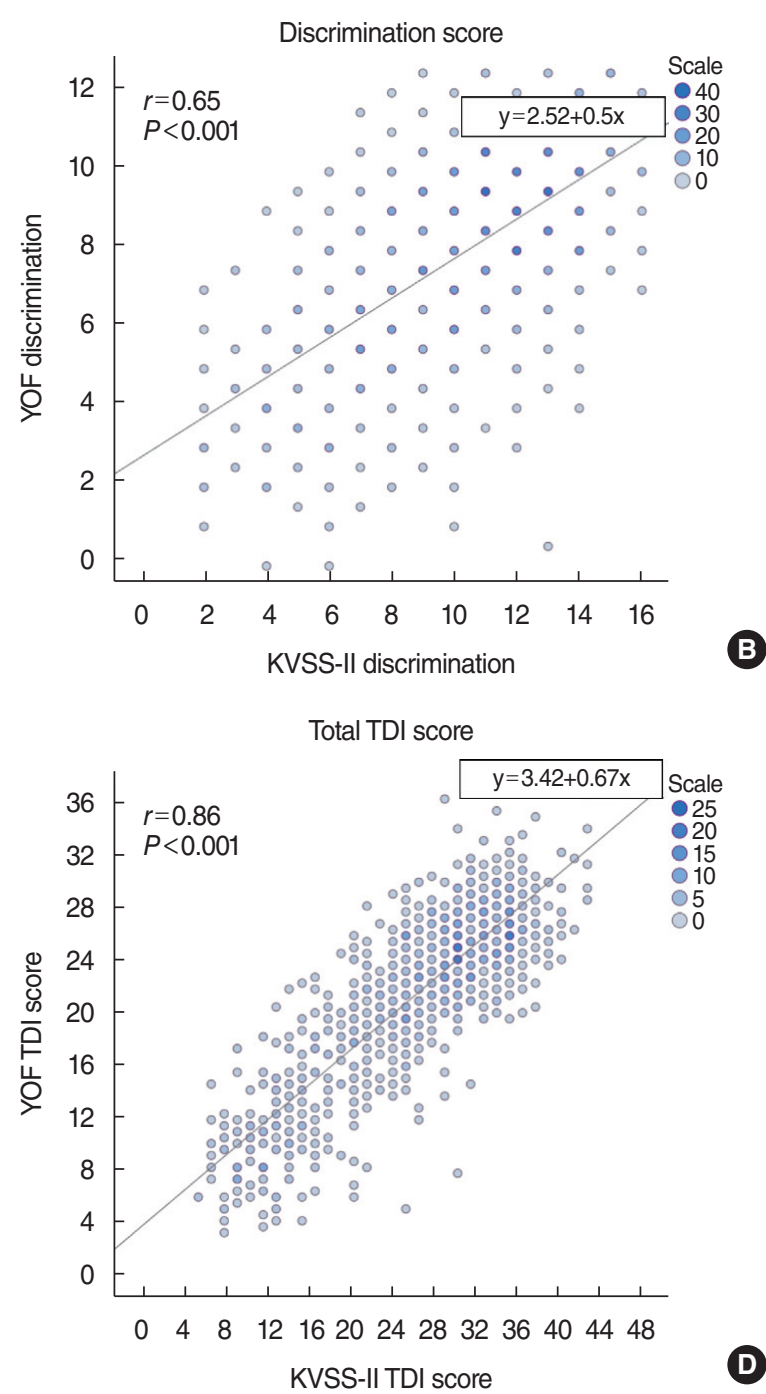

Fig. 4. Correlations between the YSK olfactory function (YOF) test and the Korean version of Sniffin' stick test (KVSS-II). (A) Threshold test, (B) discrimination test, (C) identification test, (D) total TDI score. The correlation coefficients for the YOF test and KVSS-II were 0.57 for T, 0.65 for $\mathrm{D}, 0.80$ for I, and 0.86 for the TDI score $(P<0.001$, respectively). T, threshold; D, discrimination; I, identification.

The most commonly used odorant in threshold tests is n-butanol, which has been adopted in many olfactory tests such as the Sniffin' stick test, the Connecticut Chemosensory Clinical Research Center olfactory test, and butanol threshold tests. However, as seen in the results of several animal experiments, inhalation of n-butanol may lead to neurotoxicity and developmental risks at sufficient concentrations [18-20]. The potential inhalation toxicity of n-butanol has been a focus of recent attention [21,22], indicating a need for an alternative olfactory threshold test to replace n-butanol.

PEA is another widely used odorant in olfactory threshold tests. PEA has several characteristics that are suitable for an odorant for olfactory threshold testing. First of all, it is a safe chemical, with minimal inhalation toxicity [23], and it has long been used as a raw material for flower-scented cosmetics. Secondly, PEA stimulates the trigeminal nerve less than n-butanol
[9]. The simultaneous stimulation of the trigeminal nerve along with the olfactory nerve in olfactory function test is a longstanding issue in the selection of odorants for olfactory examinations. Trigeminal stimulation can affect the detection threshold of chemicals [24], so minimizing its effect is important in establishing a reliable olfactory function test. Finally, the simple odorhedonic characteristic of PEA was considered in the choice of an odorant for the threshold test. For example, some people have good feelings about a certain odor, while others hate the same scent. If an odor has these complex hedonic properties, it may affect the olfactory test results. Pleasant and unpleasant odors are processed by different brain regions in the orbitofrontal cortex [25], causing different autonomic responses [26]. Moreover, the detection concentration of an odorant may vary depending on the environment of each individual. For example, the threshold for food flavor can be different in states of satiety 
and hunger [27]. Therefore, it can be advantageous for an odorant in an olfactory threshold test to have simple hedonic characteristics. Odor hedonics are not necessarily proportional to intensity. However, the odor-hedonic characteristics of PEA (pleasantness) and n-butanol (unpleasantness) - the two most representative odorants used in threshold tests-are simply proportional to their intensity [28]. Moreover, odor hedonics can change with concentration and intensity. Even n-butanol, which is considered to have neutral characteristics from the perspective of odor hedonics, has a change in its hedonic characteristics according to its concentration [29], whereas PEA is simply pleasant regardless of its concentration. These simple hedonic features of PEA make the results of the threshold test using PEA simple to interpret.

Comparisons of n-butanol and PEA thresholds have reported contradictory results. Croy et al. [30] reported no significant difference in thresholds between n-butanol and PEA. However, Zernecke et al. [31,32] reported conflicting results in two subsequent studies. Their findings suggested that the PEA (highest concentration, 4\%; 1:2 dilution ratio) threshold scores were significantly superior to the n-butanol (highest concentration, $4 \%$; 1:2 dilution ratio) threshold scores.

We initially considered a PEA threshold test with the highest concentration at $4 \%$, like the Sniffin' stick test. However, in the results of a preliminary study (highest concentration, $4 \% ; 1: 2$ dilution ratio; 12 steps), the olfactory threshold distribution of normosmic subjects was much lower than expected (data not shown). After adjusting the highest concentration to $10 \%$, the PEA threshold test produced a roughly normal distribution at 5-6 points, the midpoint of the 12 steps in the test (Fig. 2A).The detection threshold of n-butanol and PEA differs from study to study for several reasons. First, depending on the solvent, the detection threshold may vary, even for the same odorant [33]. The threshold test of the Sniffin' stick test, which uses n-butanol, or PEA threshold tests incorporate 16 steps of 1:2 dilutions with propylene glycol solvent, whereas the YOF test utilizes DPG, which is widely used as a solvent for cosmetics. This difference may cause a difference in the detection threshold concentration of PEA. Secondly, it is possible that the study populations differed across studies because there are no objective gold-standard criteria for normal olfactory function.

In the study, comparing the threshold test results with the 16step wide step method (i.e., the method utilized in the Sniffin' stick test) and the eight-step narrow step method demonstrated that the wide step and narrow step showed significantly reliable results, while the narrow step method showed a significantly shorter test time than the wide step method [30]. Another study investigated the reliability of reducing the number of stimuli in the threshold test using n-butanol. According to that report, using 12 steps for the threshold test had sufficient reliability, while saving test time compared to the 16-step method [34].

The KVSS-II contains odorants familiar to Koreans; however,
20 years after its development, cultural experiences have changed among generations, meaning that some odorants have become unfamiliar to Koreans. A recent study of the KVSS-II, in which the distractors were modified, reported that the correct identification percentages for normal olfactory function subjects $(n=83)$ ranged from $36 \%$ to $96 \%$ [35]. Five of the 16 odorants (leather, lemon, licorice, resin, and apple) showed a correct answer rate less than $70 \%$. These data are similar to our KVSS-II results (the percentage of correct answers ranged from $32.6 \%$ to $98.6 \%$ ), and four of the 16 odorants had a lower correct answer rate than $70 \%$ (data not shown). The odor identification task in the YOF test was designed to use Korean culture-friendly odorants. The percentage of correct answers for each odor was relatively evenly distributed (mean, 93.6\%; SD, 5.1\%; range, 80.7\%$98.6 \%$ ). According to our results, the score ratio of the identification test of the YOF test in the normosmia group was superior to that of the KVSS-II (Supplementary Fig. 1).

Generally, the molecular acceptance range of an olfactory receptor is determined by the chemical functional group. Olfactory receptors are sensitive to aldehydes or ketones, aromatic rings, or chain lengths, and so on [36]. Different odors are recognized by different combinations of olfactory receptors [37]. Therefore, if the olfactory function test can selectively provide detailed information about the major functional groups of odorants, it would be possible to interpret the results in a more complex and elaborate manner and to use them clinically. For example, if an Alzheimer disease patient shows a pattern of impaired olfactory function for a particular odorant, it may be possible that sensitivity to the major functional group of that odorant can be used to predict the likelihood of developing the disease (i.e., Alzheimer disease).

The diagnostic cutoffs for anosmia (TDI score $\leq 14.5$ ) and hyposmia $(14.5<$ TDI score $\leq 21.0)$ were obtained by calculating the Youden index, in which the maximum point indicates the point with the maximum sum of sensitivity and specificity. The Youden index of the YOF test was much higher for anosmia (0.67) than for hyposmia (0.38), suggesting that anosmia and hyposmia are more clearly distinguished by the YOF test than hyposmia and normosmia. Based on a comparison of the AUC values of the YOF test to those of the KVSS-II, the YOF test showed equivalent results to the KVSS-II in distinguishing anosmia and hyposmia.

A comparison of the YOF test and KVSS-II demonstrated that the total TDI score and the respective subtest scores for threshold, discrimination, and identification were closely correlated. The Pearson correlation coefficient of the total TDI scores between the YOF test and KVSS-II was higher than that of each T/ D/I subtest. Furthermore, on the YOF test for anosmia determination, the AUC value of the total TDI score was higher than that of each T/D/I subtest (data not shown). As described above, threshold detection, discrimination, and odor information might be affected by different levels of cognition/memory [6]. Taking these results into account, analyzing the total TDI score can pro- 
vide more comprehensive and accurate information about individual olfactory function than analyzing each T/D/I subtest separately [38].

In conclusion, we developed an olfactory test that uses safe and Korean culture-friendly odorants. Various olfactory characteristics were reliably evaluated using the YOF test and demonstrated validity in comparison to another widely used olfactory function test. The YOF test is expected to provide a sufficiently comprehensive olfactory assessment that saves time compared to conventional tests. Thus, we could conclude that the YOF test proved useful to identify the olfactory function of Koreans. Further studies are being conducted to identify the safety of the odorants of the YOF test and to determine whether the sensitivity of major functional groups varies depending on the etiology of olfactory loss.

\section{CONFLICT OF INTEREST}

No potential conflict of interest relevant to this article was reported.

\section{ACKNOWLEDGMENTS}

This research was supported by the Bio \& Medical Technology Development Program of the National Research Foundation (NRF) funded by the Ministry of Science, ICT \& Future Planning (NRF-2016M3A9D5A01952414). This work was also supported by a National Research Foundation of Korea (NRF) grant funded by the Korean government (MSIP) (No. 2016R1A5A2008630).

\section{ORCID}

Jong-Gyun Ha Jinwon Kim Jae Sung Nam Jeong Jin Park Hyung-Ju Cho Joo-Heon Yoon Chang-Hoon Kim https://orcid.org/0000-0002-2712-1297 https://orcid.org/0000-0001-9144-2979 https://orcid.org/0000-0002-3428-8108 https://orcid.org/0000-0002-1160-5713 https://orcid.org/0000-0002-2851-3225 https://orcid.org/0000-0003-2404-7156 https://orcid.org/0000-0003-1238-6396

\section{AUTHOR CONTRIBUTIONS}

Conceptualization: JHY, CHK. Data curation: JGH, JK, JSN, JJP. Formal analysis: JGH. Funding acquisition: CHK. Methodology: JGH, HJC, JHY, CHK. Visualization: JGH. Writing-original draft: JGH, HJC. Writing-review \& editing: HJC, JHY, CHK.

\section{SUPPLEMENTARY MATERIALS}

Supplementary materials can be found via https://doi.org/10. 21053/ceo.2020.00864.

\section{REFERENCES}

1. Blomqvist EH, Bramerson A, Stjarne P, Nordin S. Consequences of olfactory loss and adopted coping strategies. Rhinology. 2004 Dec; 42(4):189-94.

2. Asahina K, Pavlenkovich V, Vosshall LB. The survival advantage of olfaction in a competitive environment. Curr Biol. 2008 Aug;18(15): 1153-5.

3. Berendse HW, Ponsen MM. Detection of preclinical Parkinson's disease along the olfactory trac(t). J Neural Transm Suppl. 2006;(70): 321-5.

4. Jung HJ, Shin IS, Lee JE. Olfactory function in mild cognitive impairment and Alzheimer's disease: a meta-analysis. Laryngoscope. 2019 Feb;129(2):362-9.

5. Pause BM, Miranda A, Goder R, Aldenhoff JB, Ferstl R. Reduced olfactory performance in patients with major depression. J Psychiatr Res. 2001 Sep-Oct;35(5):271-7.

6. Hedner M, Larsson M, Arnold N, Zucco GM, Hummel T. Cognitive factors in odor detection, odor discrimination, and odor identification tasks. J Clin Exp Neuropsychol. 2010 Dec;32(10):1062-7.

7. Hsieh JW, Keller A, Wong M, Jiang RS, Vosshall LB. SMELL-S and SMELL-R: olfactory tests not influenced by odor-specific insensitivity or prior olfactory experience. Proc Natl Acad Sci U S A. 2017 Oct; 114(43):11275-84.

8. Hummel T, Whitcroft KL, Andrews P, Altundag A, Cinghi C, Costanzo RM, et al. Position paper on olfactory dysfunction. Rhinology. 2016 Jan;56(1):1-30.

9. Doty RL, BruggerWE, Jurs PC, Orndorff MA, Snyder PJ, Lowry LD. Intranasal trigeminal stimulation from odorous volatiles: psychometric responses from anosmic and normal humans. Physiol Behav. 1978 Feb;20(2):175-85.

10. Doty RL, Smith R, McKeown DA, Raj J. Tests of human olfactory function: principal components analysis suggests that most measure a common source of variance. Percept Psychophys. 1994 Dec;56(6): 701-7.

11. Rospars JP. Interactions of odorants with olfactory receptors and other preprocessing mechanisms: how complex and difficult to predict? Chem Senses. 2013 May;38(4):283-7.

12. Hong SC, Yoo YS, Kim ES, Kim SC, Park SH, Kim JK, et al. Development of KVSS test (Korean version of Sniffin' Sticks Test). Korean J Otolaryngol-Head Neck Surg. 1999 Jul;42(7):855-60.

13. HummelT, Sekinger B, Wolf SR, Pauli E, Kobal G. 'Sniffin' sticks': olfactory performance assessed by the combined testing of odor identification, odor discrimination and olfactory threshold. Chem Senses. 1997 Feb;22(1):39-52.

14. Hong SM, Park IH, Kim KM, Shin JM, Lee HM. Relationship between the Korean version of the Sniffin' Stick Test and the T\&T Olfactometer in the Korean population. Clin Exp Otorhinolaryngol. 2011 Dec; 4(4):184-7.

15. Cho JH, Jeong YS, Lee YJ, Hong SC, Yoon JH, Kim JK. The Korean version of the Sniffin' stick (KVSS) test and its validity in comparison with the cross-cultural smell identification test (CC-SIT). Auris Nasus Larynx. 2009 Jun;36(3):280-6.

16. Youden WJ. Index for rating diagnostic tests. Cancer. 1950 Jan;3(1): 32-5.

17. DeLong ER, DeLong DM, Clarke-Pearson DL. Comparing the areas 
under two or more correlated receiver operating characteristic curves: a nonparametric approach. Biometrics. 1988 Sep;44(3):837-45.

18. Hackett PL, Brown MG, Buschbom RL, Clark ML, Miller RA, Music $\mathrm{RL}$, et al. Teratogenic study of ethylene and propylene oxide and nbutyl acetate. Richland (WA): Battelle Pacific Northwest Laboratories; 1982.

19. David RM, Tyler TR, Ouellette R, FaberWD, Banton MI. Evaluation of subchronic toxicity of n-butyl acetate vapor. Food Chem Toxicol. 2001 Aug;39(8):877-86.

20. Saillenfait AM, Gallissot F, Sabate JP, Bourges-Abella N, Muller S. Developmental toxic effects of ethylbenzene or toluene alone and in combination with butyl acetate in rats after inhalation exposure. $\mathrm{J}$ ApplToxicol. 2007 Jan-Feb;27(1):32-42.

21. Bale AS, Lee JS. An overview of butanol-induced developmental neurotoxicity and the potential mechanisms related to these observed effects. Neurotoxicol Teratol. 2016 Jan-Feb;53:33-40.

22. Segal D, Bale AS, Phillips LJ, Sasso A, Schlosser PM, Starkey C, et al. Issues in assessing the health risks of n-butanol. J Appl Toxicol. 2020 Jan;40(1):72-86.

23. Scognamiglio J, Jones L, Letizia CS, Api AM. Fragrance material review on phenylethyl alcohol. Food Chem Toxicol. 2012 Sep;50 Suppl 2:S224-39.

24. Jacquot L, Hidalgo J, Brand G. Just noticeable difference in olfaction is related to trigeminal component of odorants. Rhinology. 2010 Sep; 48(3):281-4.

25. Grabenhorst F, Rolls ET, Margot C, da Silva MA, Velazco MI. How pleasant and unpleasant stimuli combine in different brain regions: odor mixtures. J Neurosci. 2007 Dec;27(49):13532-40.

26. Bensafi M, Rouby C, Farget V, Bertrand B, Vigouroux M, Holley A. Autonomic nervous system responses to odours: the role of pleasantness and arousal. Chem Senses. 2002 Oct;27(8):703-9.

27. Albrecht J, Schreder T, Kleemann AM, Schopf V, Kopietz R, Anzinger A, et al. Olfactory detection thresholds and pleasantness of a foodrelated and a non-food odour in hunger and satiety. Rhinology. 2009 Jun;47(2):160-5.
28. Doty RL.An examination of relationships between the pleasantness, intensity, and concentration of 10 odorous stimuli. Percept Psychophys. 1975 Sep;17:492-6.

29. Hong SJ, Li H, Kim ST. Semantic differential evaluation of n-Butanol odor profile: suitability as odor intensity reference. J Odor Indoor Environ. 2019 Jun;18(2):102-11.

30. Croy I, Lange K, Krone F, Negoias S, Seo HS, Hummel T. Comparison between odor thresholds for phenyl ethyl alcohol and butanol. Chem Senses. 2009 Jul;34(6):523-7.

31. Zernecke R, Vollmer B, Albrecht J, Kleemann AM, Haegler K, Linn J, et al. Comparison of two different odorants in an olfactory detection threshold test of the Sniffin' Sticks. Rhinology. 2010 Sep;48(3):36873.

32. Zernecke R, FrankT, Haegler K, Albrecht J, Bruckmann H, Wiesmann M. Correlation analyses of detection thresholds of four different odorants. Rhinology. 2011 Aug;49(3):331-6.

33. Tsukatani T, Miwa T, Furukawa M, Costanzo RM. Detection thresholds for phenyl ethyl alcohol using serial dilutions in different solvents. Chem Senses. 2003 Jan;28(1):25-32.

34. Kern DW, Schumm LP, Wroblewski KE, Pinto JM, Hummel T, McClintock MK. Olfactory thresholds of the U.S. population of homedwelling older adults: development and validation of a short, reliable measure. PLoS One. 2015 Mar;10(3):e0118589.

35. Kim JM, Jeong MS, Shin DH, Seol JH, Hong SC, Cho JH, et al. Olfactory identification test using familiar distracters for Koreans. Clin Exp Otorhinolaryngol. 2014 Mar;7(1):19-23.

36. Poivet E, Tahirova N, Peterlin Z, Xu L, Zou DJ, Acree T, et al. Functional odor classification through a medicinal chemistry approach. Sci Adv. 2018 Feb;4(2):eaao6086.

37. Malnic B, Hirono J, Sato T, Buck LB. Combinatorial receptor codes for odors. Cell. 1999 Mar;96(5):713-23.

38. Lotsch J, Reichmann H, Hummel T. Different odor tests contribute differently to the evaluation of olfactory loss. Chem Senses. 2008 Jan;33(1):17-21. 